\title{
Impact of COVID-19 on routine malaria indicators in rural Uganda: an interrupted time series analysis
}

\author{
Jane F. Namuganga ${ }^{1 *} \mathbb{C}$, Jessica Briggs ${ }^{2}$, Michelle E. Roh ${ }^{3}$, Jaffer Okiring ${ }^{1}$, Yasin Kisambira' ${ }^{1}$ Asadu Sserwanga ${ }^{1}$, \\ James A. Kapisi' , Emmanuel Arinaitwe' ${ }^{1}$, Chris Ebong ${ }^{1}$, Isaac Ssewanyana' , Catherine Maiteki-Ssebuguzi ${ }^{4}$, \\ Moses R. Kamya ${ }^{1,5}$, Sarah G. Staedke ${ }^{6}$, Grant Dorsey ${ }^{2}$ and Joaniter I. Nankabirwa ${ }^{1,5}$
}

\begin{abstract}
Background: In March 2020, the government of Uganda implemented a strict lockdown policy in response to the COVID-19 pandemic. Interrupted time series analysis (ITSA) was performed to assess whether major changes in outpatient attendance, malaria burden, and case management occurred after the onset of the COVID-19 epidemic in rural Uganda.

Methods: Individual level data from all outpatient visits collected from April 2017 to March 2021 at 17 facilities were analysed. Outcomes included total outpatient visits, malaria cases, non-malarial visits, proportion of patients with suspected malaria, proportion of patients tested using rapid diagnostic tests (RDTs), and proportion of malaria cases prescribed artemether-lumefantrine (AL). Poisson regression with generalized estimating equations and fractional regression was used to model count and proportion outcomes, respectively. Pre-COVID trends (April 2017-March 2020) were used to predict the'expected' trend in the absence of COVID-19 introduction. Effects of COVID-19 were estimated over two six-month COVID-19 time periods (April 2020-September 2020 and October 2020-March 2021) by dividing observed values by expected values, and expressed as ratios.

Results: A total of 1,442,737 outpatient visits were recorded. Malaria was suspected in $55.3 \%$ of visits and $98.8 \%$ of these had a malaria diagnostic test performed. ITSA showed no differences between observed and expected total outpatient visits, malaria cases, non-malarial visits, or proportion of visits with suspected malaria after COVID-19 onset. However, in the second six months of the COVID-19 time period, there was a smaller mean proportion of patients tested with RDTs compared to expected (relative prevalence ratio $(\mathrm{RPR})=0.87, \mathrm{Cl}(0.78-0.97)$ ) and a smaller mean proportion of malaria cases prescribed $\mathrm{AL}(\mathrm{RPR}=0.94, \mathrm{Cl}(0.90-0.99)$ ).

Conclusions: In the first year after the COVID-19 pandemic arrived in Uganda, there were no major effects on malaria disease burden and indicators of case management at these 17 rural health facilities, except for a modest decrease in the proportion of RDTs used for malaria diagnosis and the mean proportion of malaria cases prescribed AL in the second half of the COVID-19 pandemic year. Continued surveillance will be essential to monitor for changes in trends in malaria indicators so that Uganda can quickly and flexibly respond to challenges imposed by COVID-19.
\end{abstract}

*Correspondence: jnamuganga@idrc-uganda.org

${ }^{1}$ Infectious Diseases Research Collaboration, Kampala, Uganda

Full list of author information is available at the end of the article

\section{Background}

Significant progress in malaria control has been realized in sub-Saharan Africa over the last two decades following the scale-up of effective malaria control interventions original author(s) and the source, provide a link to the Creative Commons licence, and indicate if changes were made. The images or other third party material in this article are included in the article's Creative Commons licence, unless indicated otherwise in a credit line to the material. If material is not included in the article's Creative Commons licence and your intended use is not permitted by statutory regulation or exceeds the permitted use, you will need to obtain permission directly from the copyright holder. To view a copy of this licence, visit http://creativecommons.org/licenses/by/4.0/. The Creative Commons Public Domain Dedication waiver (http://creativeco mmons.org/publicdomain/zero/1.0/) applies to the data made available in this article, unless otherwise stated in a credit line to the data. 
[1]. At the start of the COVID-19 pandemic, there was concern that progress in the fight against malaria would be reversed due to interruption of malaria control interventions and overwhelmed health care systems, with some modelling studies suggesting that malaria morbidity and /or mortality may double due to COVID-19 [2-4]. In Uganda, malaria is endemic in over $95 \%$ of the country and the leading cause of morbidity and mortality, accounting for $30-50 \%$ of outpatient visits, $15-20 \%$ of all hospital admissions, and up to $20 \%$ of all hospital deaths [5]. While successes have been registered in malaria control in the country in the last decade, including increased coverage of control interventions and reductions in the overall disease burden $[6,7]$, there is concern that these achievements may be reversed by the global COVID-19 pandemic.

The World Health Organization (WHO) declared COVID-19 a global pandemic on 11 March 2020 [8]. The Government of Uganda swiftly implemented strict restrictions and diverted personnel and resources to minimize the spread of SARS-CoV-2 and mitigate the economic burden of the pandemic. On 18 March, 2020, mass gatherings were suspended, and a 14-day quarantine was imposed on all travellers arriving in Uganda [8]. When the country registered its first confirmed case on $21^{\text {st }}$ March 2020, additional restrictions were implemented, including: (1) closure of borders except for cargo and goods on 21 March, 2020; (2) suspension of public transport and restrictions on movement of private vehicle on 25 March, 2020; (3) mandatory testing of truck drivers on 10 April, 2021; (4) a national lockdown and curfew from 19.00 to 06.30 on 30 March, 2020, initially for 14 days but eventually extended to 26 May, 2020, and (5) closure of all schools [8]. Despite these measures, Uganda's COVID19 cases progressively increased over the period with the country registering its first 100 confirmed cases on 6 May, 2020, first death on 23 July, 2020, first 1000 cases on 9 June, 2020 and first wave peak in December 2020.

Although lockdowns have been shown to minimize the spread of COVID-19 [9-11], they may have major repercussions on malaria treatment and prevention including decreased access to health care, interruption of service delivery, and disruption of delivery of malaria control interventions such as long-lasting insecticide treated net (LLIN) or indoor residual spraying (IRS) campaigns. In addition, an increase in the incidence of fever cases due to COVID-19 may lead to an increase in the number of suspected malaria cases presenting at health facilities, increasing demand for malaria diagnostics, and potentially leading to over diagnosis and overtreatment of malaria and/or a shortage of diagnostics. Indeed, a modelling study by WHO predicted that in the 'worst case' scenario where LLINs campaigns were cancelled and access to effective antimalarial treatment was severely disrupted, malaria deaths could increase by $>200 \%$ in Uganda as result of the COVID-19 pandemic [12].

Interrupted time series analysis (ITSA) is a quasiexperimental study design that can be used to estimate causal effects using observational data. It is often used to evaluate the effectiveness of population-level health interventions that have been implemented at a clearly defined point in time [13-16]. ITSA has also been used to evaluate the effect of an unplanned event on health outcomes, such as the effect of the late 2000s financial crisis on suicides in Spain [17]. A key assumption of ITSA is that the pre-intervention trend would provide a suitable estimate of the post-intervention trend had the intervention never occurred. In this study, interrupted time series analysis was used to assess the effect of the COVID-19 pandemic in Uganda on malaria disease burden and case management at 17 public health facilities across rural Uganda using three years of pre-COVID data to model the baseline trend. Results from the study will improve our understanding of the effect of COVID-19 on malaria care in Uganda and in other countries with similar settings.

\section{Methods}

\section{Health-facility based surveillance}

The study utilized data collected at 17 Malaria Reference Centres (MRCs) located in rural settings from April 2017 through March 2021 (Fig. 1). MRCs are high-volume, level III/IV, public health facilities located throughout Uganda in areas with varying malaria transmission intensities. MRCs were first established in 2006 by the Uganda Malaria Surveillance Project (UMSP), a project led by Makerere University, University of California, San Francisco (UCSF), and Infectious Diseases Research Collaboration (IDRC) groups, in collaboration with the Uganda Ministry of Health. Details of the MRCs have been described elsewhere [18] but briefly, there are currently 70 MRCs collecting high quality, individual level data from all patients presenting at the outpatient clinics of the facilities. Of the 70 MRCs, 17 were selected for this analysis based on the following criteria: (1) MRC was opened and data collection commenced before January 2017; and (2) MRC had less than 5\% missing data on four of the variables (suspected malaria case, malaria test done, malaria test result recorded, type of malaria test done).

At each MRC, patient data are recorded in standardized health management information system (HMIS) registers (HMIS 002-outpatient register) at the outpatient clinics. Data are transcribed from the registers into Microsoft Access databases by on-site records officers. Data collected include patient demographics, 


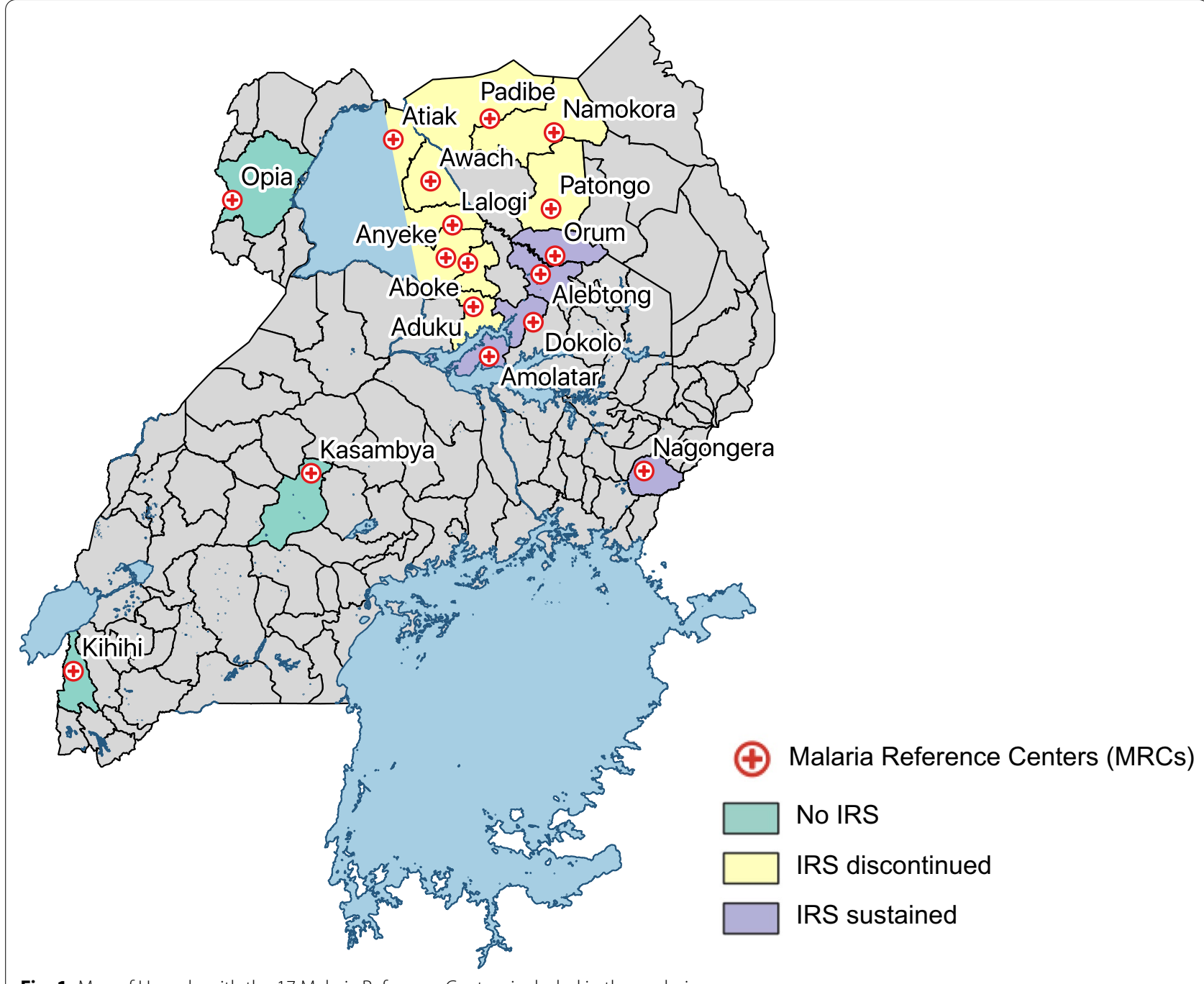

Fig. 1 Map of Uganda with the 17 Malaria Reference Centres included in the analysis

village of residence, history of fever, whether a malaria diagnostic test was performed, type of malaria test done (rapid diagnostic test (RDT) vs microscopy), results of laboratory tests, diagnoses given, and treatments prescribed. Data are not collected on whether a patient receives or is adherent to the prescribed treatment. On average, over 1,400 patient visits and 1,000 suspected malaria cases tested are captured monthly at each MRC. Adherence to malaria case management guidelines is emphasized and supported at the MRCs through training, onsite mentorship and support from regional surveillance assistants, regular continuous medical education sessions, and monthly data analysis and feedback sessions for on-site health workers. Routine data quality assessments are undertaken at each site to ensure collection of high quality data in terms of completeness and accuracy.

\section{Laboratory procedures}

All patients suspected to have malaria are routinely sent to an onsite laboratory for confirmatory testing using either malaria microscopy or malaria RDT kits. Malaria microscopy at the MRCs is conducted by skilled laboratory personnel and generally reserved for when RDTs are not available or of limited stock and upon request from a clinician. Quality control for malaria microscopy is conducted monthly at each site by a laboratory team who are part of the core UMSP team. They also provide onsite mentorship for site laboratory personnel to ensure high quality malaria testing.

\section{Malaria control interventions}

Malaria control activities at the MRCs during the study period included: (1) malaria case management as per the Uganda National Treatment Guidelines [19] which 
recommends artemether-lumefantrine (AL)as first-line therapy; (2) promotion of intermittent preventive treatment during pregnancy; and 3) universal distribution of free LLINs. Two national LLIN distribution campaigns were conducted during the study period, in 2017-18 and 2020-2021 (Figs. 1 and 2). The status of IRS campaigns varied across the sites included in this study as shown in Fig. 2; three sites were in districts that never received IRS; IRS was discontinued in 2017 in districts where nine sites were located, and the remaining five sites were in districts that had sustained IRS campaigns throughout the study period.

\section{Statistical analysis}

ITSA, using segmented regression, were conducted to estimate the impact of the COVID-19 epidemic on key malaria indicators. Data from all 17 sites from April 2017 through the end of March 2021 were included in the analysis. The study period included three years pre-COVID and one year after the arrival of COVID-19 in Uganda, with the start of the COVID-19 time period defined as 1 April, 2020 (Fig. 2). Outcomes for the ITSA included number of total outpatient visits, number of non-malarial visits (visits in which malaria was not diagnosed), malaria visits (visits in which a laboratory-confirmed diagnosis of malaria was made, synonymous with malaria cases), proportion of all patient visits with suspected malaria, proportion of patients tested using RDTs, and proportion of patients with confirmed malaria prescribed AL.

In the segmented regression model, the baseline trend during the pre-COVID-19 period and the slope change following the arrival of COVID-19 in the country are modelled. To estimate the counterfactual (COVID19 time period) trend, the slope change is set to 0 . The generalized segmented regression model is as follows: $Y_{h t}=\beta_{0}+\beta_{1} T+\beta_{2} P_{t}+\beta_{3} C_{t}+\beta_{4} R_{h t}$, where $Y_{h t}$ represents the outcome recorded at the health facility $h$ in month $\mathrm{t}, \beta_{0}$ is the intercept at the start of the study $(\mathrm{t}=0)$, $\mathrm{T}$ is a linear term indicating the time in months since the start of the study observation period (models the preCOVID trend), $P_{t}$ is a linear term indicating the months since the start of the COVID-19 time period (models the observed change in trend after COVID onset), $C_{t}$ is a linear term indicating calendar month fixed effects (e.g., January, February, etc.) to model seasonality), and $R_{h t}$ is a vector of monthly rainfall data $(\mathrm{mm})$ lagged by one month and averaged across the district-level. Poisson regression was used to model count outcomes using a generalized estimating equation (to account for clustered observations between sites) with an autoregressive of order one (ar1) correlation structure (to account for serial autocorrelation of error terms of outcomes of adjacent time periods within-sites). Fractional regression was used to model proportional outcomes.

For ITSA models that included malaria outcomes, additional linear terms representing the number of months since LLIN distribution and the round of IRS were included to adjust for time-varying confounding. Based on surveillance data from these sites, LLIN campaigns were assumed to confer 24 months of protection, whereas IRS campaigns (which used Actellic or Sumishield-based insecticides) were assumed to provide up to a year of protection. For Poisson models, the underlying population was assumed constant throughout the study period and thus no offset term was included.

Models were then used to predict the unobserved counterfactual values (i.e., 'expected' values in absence of COVID-19) for each health facility in each month (i.e., monthly number of events or proportions had the epidemic not occurred) by setting $P_{t}$ to zero. For count outcomes, incidence rate ratios (IRR) were calculated by summing the outcomes across the first and second six months after COVID-19 started in Uganda and dividing the observed by the expected values. For

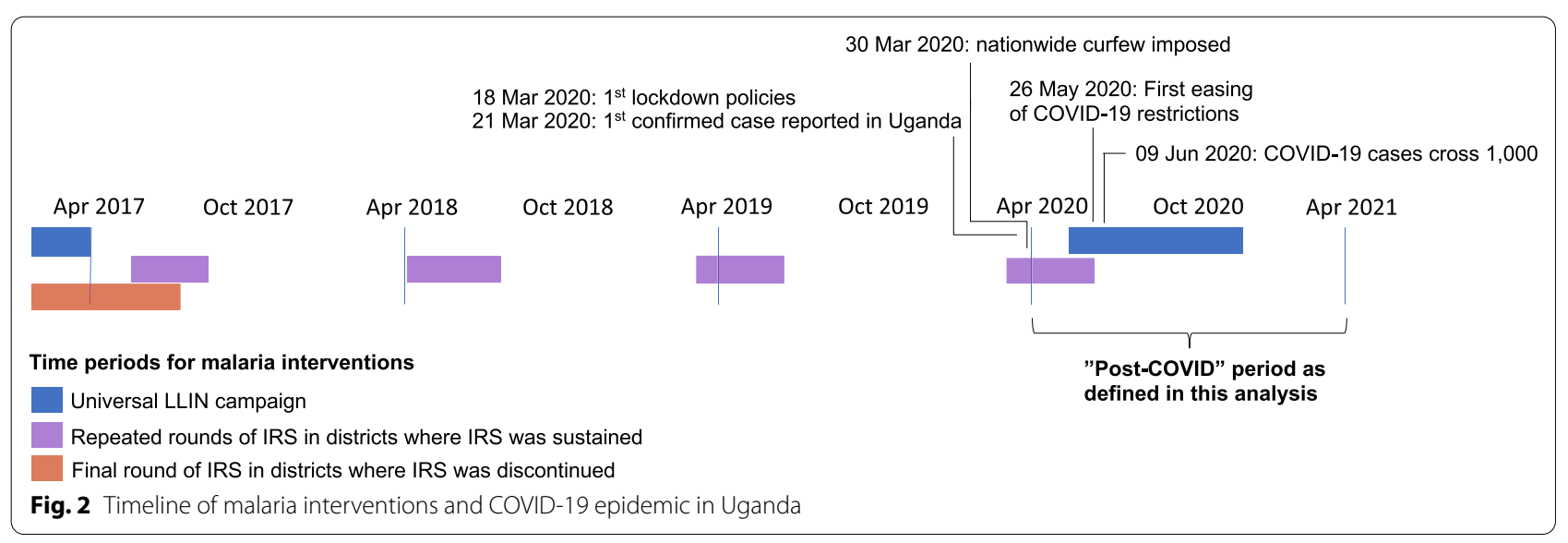


proportion outcomes, relative prevalence ratios (RPR) were calculated by averaging the monthly proportions across the two six-month COVID-19 time periods and dividing the observed by expected values. 95\% confidence intervals were obtained using a 1,000 block, percentile-bootstrapping procedure, where sites were resampled with replacement.

\section{Results}

From April 2017 through March 2021, 1,442,737 patient visits were recorded at the 17 MRCs (Table 1). The median age of patients was 23 years, and the majority (67\%) were female. Malaria was suspected in 798,270 (55.3\%) of all patient visits, and almost all (98.8\%) patients with suspected malaria had a malaria diagnostic test done. RDTs were the most common laboratory diagnostic tool used to test patients for malaria (83.8\%), although this varied by site (range: 24.0 to $99.8 \%$ ). Overall, malaria test positivity rate was $53.1 \%$, ranging from 14.1 to $69.8 \%$ at individual MRCs. Most patients with confirmed malaria were prescribed AL (93\%), the recommended first line treatment for uncomplicated malaria in Uganda. A detailed description of the study population stratified by site and status of IRS is presented in Table 1.

\section{Trends in disease burden and case management} over the study period

Over the four-year study period, a single annual peak was observed in the number of monthly outpatient visits, with the highest number of visits observed during midyear of 2019 (>2,500 visits, Fig. 3A). The monthly trend in the number of outpatient visits for non-malarial illness was declining at a steady pace before the COVID-19 epidemic (Fig. 3B). The number of malaria visits followed a similar trend to that of the total outpatient visits, with peaks in these occurring simultaneously (Fig. 3C). Similar trends were observed with the proportion of suspected malaria cases (Fig. 3D). The proportion of patients tested with RDT each month remained consistently high $(>70 \%)$ and almost constant throughout the study period, except in 2018 when the proportion reached $>90 \%$ and in 2021 when the proportion fell below 70\% (Fig. 3E). The proportion of confirmed malaria cases prescribed AL (Fig. 3F) was consistently high, remaining $>90 \%$ from late 2019 through 2020, before a decline was observed in 2021. The mean proportion of patients with suspected malaria who received a diagnostic test is not shown in Fig. 3 because it remained consistently high across all study time points (Additional file 1: Fig. S1) and therefore a formal ITSA was not performed.

Table 1 Cumulative totals of key malaria indicators stratified by malaria reference centre (MRC) from April 2017 to March 2021

\begin{tabular}{|c|c|c|c|c|c|c|c|c|c|}
\hline IRS status & MRC & $\begin{array}{l}\text { Total } \\
\text { number of } \\
\text { outpatient } \\
\text { visits }\end{array}$ & $\begin{array}{l}\text { Median } \\
\text { age in } \\
\text { years }\end{array}$ & $\begin{array}{l}\text { Proportion } \\
\text { female }\end{array}$ & $\begin{array}{l}\text { Malaria } \\
\text { suspected } \\
\text { (\% total } \\
\text { visits) }\end{array}$ & $\begin{array}{l}\text { Tested for } \\
\text { malaria } \\
\text { (\% malaria } \\
\text { suspected) }\end{array}$ & $\begin{array}{l}\text { RDT } \\
\text { performed } \\
\text { (\% tested for } \\
\text { malaria) }\end{array}$ & $\begin{array}{l}\text { Laboratory } \\
\text { confirmed } \\
\text { malaria (\% } \\
\text { tested for } \\
\text { malaria) }\end{array}$ & $\begin{array}{l}\text { AL } \\
\text { prescribed* } \\
\text { (\% laboratory } \\
\text { confirmed } \\
\text { malaria) }\end{array}$ \\
\hline \multirow{3}{*}{$\begin{array}{l}\text { No recent } \\
\text { history }\end{array}$} & Kasambya & 59,574 & 20.7 & 67.5 & $44,118(74.1)$ & $42,830(97.1)$ & $24,522(57.3)$ & $19,100(44.6)$ & $18,801(98.4)$ \\
\hline & Kihihi & 79,561 & 25.2 & 65.6 & $46,207(58.1)$ & $46,153(99.9)$ & $11,061(24.0)$ & $17,322(37.5)$ & $16,099(92.9)$ \\
\hline & Opia & 57,879 & 17.7 & 63.6 & $46,586(80.5)$ & $46,335(99.5)$ & $46,270(99.8)$ & $28,378(61.2)$ & $28,091(99.0)$ \\
\hline \multirow{9}{*}{$\begin{array}{l}\text { IRS discontin- } \\
\text { ued }\end{array}$} & Aduku & 102,761 & 24.7 & 69.9 & $58,404(56.8)$ & $57,157(97.9)$ & $36,702(64.2)$ & $31,723(55.5)$ & $30,910(97.4)$ \\
\hline & Anyeke & 108,612 & 24.6 & 66.3 & $52,820(48.6)$ & $51,765(98.0)$ & $46,143(89.1)$ & $35,056(67.7)$ & $32,089(91.5)$ \\
\hline & Aboke & 81,626 & 24.2 & 68.6 & $50,075(61.3)$ & $49,740(99.3)$ & $45,908(92.3)$ & $34,696(69.8)$ & $32,920(94.9)$ \\
\hline & Awach & 110,328 & 20.7 & 69.2 & $61,252(55.5)$ & $59,357(96.9)$ & $58,183(98.0)$ & $38,343(64.6)$ & $35,956(93.8)$ \\
\hline & Lalogi & 110,585 & 21.8 & 68.7 & $67,335(60.9)$ & 67,095 (99.6) & $65,652(97.8)$ & $38,572(57.5)$ & 35,315 (91.6) \\
\hline & Patongo & 77,556 & 20.4 & 66.2 & $54,394(70.1)$ & $53,794(98.9)$ & $51,244(95.3)$ & $29,858(55.5)$ & $28,619(95.9)$ \\
\hline & Atiak & 87,872 & 19.8 & 65.5 & 49,529 (56.4) & $48,685(98.3)$ & 48,477 (99.6) & $32,894(67.6)$ & $30,530(92.8)$ \\
\hline & Padibe & 89,262 & 21.3 & 66.5 & $56,985(63.8)$ & $56,849(99.8)$ & $55,864(98.3)$ & $35,023(61.6)$ & $32,484(92.8)$ \\
\hline & Namokora & 92,605 & 20.6 & 65.0 & $64,242(69.4)$ & $63,930(99.5)$ & $57,983(90.7)$ & $36,163(56.5)$ & $32,216(89.2)$ \\
\hline \multirow[t]{5}{*}{ IRS sustained } & Nagongera & 77,735 & 23.3 & 65.8 & 27,667 (35.6) & $27,623(99.8)$ & $14,286(51.7)$ & $3,891(14.1)$ & $3,459(88.9)$ \\
\hline & Amolatar & 71,440 & 26.5 & 65.8 & $19,231(26.9)$ & $19,132(99.5)$ & $16,302(85.2)$ & $6173(32.3)$ & $5417(87.8)$ \\
\hline & Dokolo & 115,297 & 27.1 & 68.6 & $42,046(36.5)$ & $41,847(99.5)$ & $38,555(92.1)$ & $13,124(31.4)$ & $11,617(88.5)$ \\
\hline & Orum & 47,440 & 26.5 & 65.5 & $26,265(55.4)$ & $26,252(100)$ & $16,128(61.4)$ & $10,162(38.7)$ & $7,799(76.7)$ \\
\hline & Alebtong & 72,604 & 27.8 & 70.1 & $31,114(42.9)$ & $30,424(97.8)$ & 28,173 (92.6) & $8,379(27.5)$ & $7,726(92.2)$ \\
\hline \multicolumn{2}{|c|}{ All sites combined } & $1,442,737$ & 23.0 & 67.0 & $798,270(55.3)$ & $788,988(98.8)$ & $661,453(83.8)$ & $418,830(53.1)$ & $390,048(93.1)$ \\
\hline
\end{tabular}



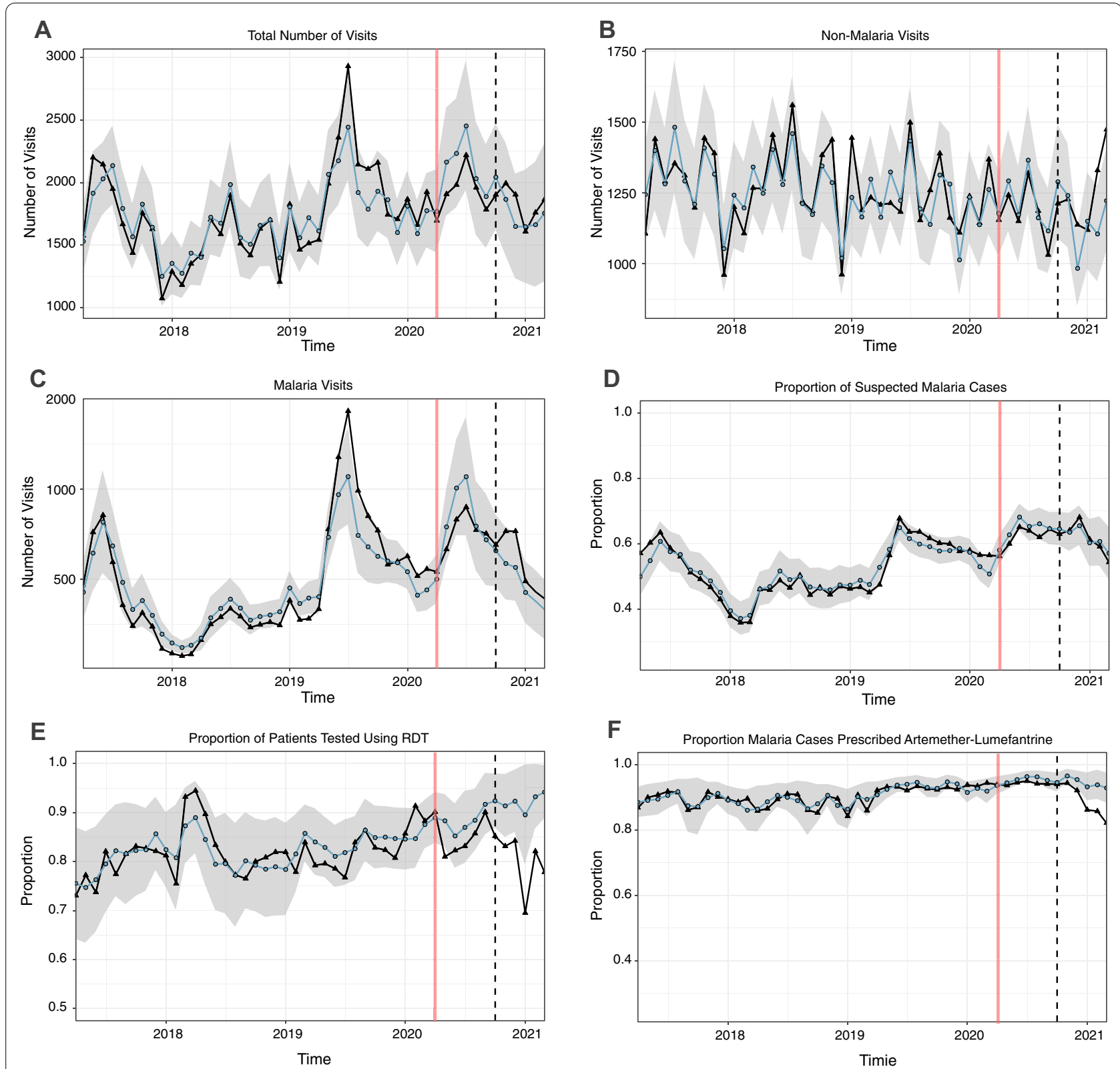

$\rightarrow$ Observed $\triangle$ Predicted

Fig. 3 Observed and expected $\mathbf{A}$ total number of visits, B non-malaria visits, $\mathbf{C}$ visits where malaria was diagnosed, $\mathbf{D}$ mean proportion of suspected malaria cases, and $\mathbf{E}$ mean proportion of patients tested using RDT by month, and $\mathbf{F}$ mean proportion of malaria cases prescribed artemether-lumefantrine. The grey ribbon represents the bootstrapped $95 \%$ confidence interval of the model. Vertical red line represents the start of the COVID-19 time-period on 1 April 2020. Vertical black dashed line represents the 6-month midpoint of the COVID-19 time period (1 October, 2020)

\section{Impact of COVID-19 on disease burden and case management}

Figure 3 shows that, for all outcomes except proportion of patients tested with RDT and proportion of malaria cases prescribed AL, there was no statistically significant change in the 12-month COVID-19 time period (April
2020- March 2021); observed monthly values fell within the $95 \%$ confidence interval of the expected values. This held true for total outpatient visits, malaria and nonmalaria visits, and proportion of patients suspected to have malaria. However, for proportion of patients tested with RDTs and proportion of malaria cases prescribed 
AL, observed values were lower than expected in the last six months of observation based on pre-COVID trends. Effect estimates across all measured outcomes are presented in Table 2, stratified into the first and second six months of the COVID-19 time intervals. In the first six months after the onset of COVID-19 epidemic, during which the lockdown was strictest, there were no significant differences in the observed versus expected numbers of total visits $(196,300$ vs 216,822 ; IRR $=0.91$ (0.82-1.00)), malaria cases $(75,825$ vs 82,$884 ;$ IRR $=0.92$ $(0.76-1.14))$, and non-malarial visits $(120,475$ vs 123,902 ; IRR $=0.97(0.92-1.04))$. There were also no significant differences in the mean proportion of suspected malaria cases, mean proportion tested with RDT, or mean proportion prescribed AL.

Over the second six months of the COVID-19 time period (October 2020-March 2021), during which lockdown restrictions were eased despite increasing COVID19 cases in the country, there were again no significant differences observed versus expected numbers total visits $[187,573$ vs 186,136 ; RR $1.01(0.81-1.23)]$, malaria cases $[60,032$ vs 50,050 , RR $1.20(0.75,1.84)]$ and nonmalarial visits [127,541 vs 118,890; RR 1.07 (0.95-1.21)]. There was also no difference in the proportion of suspected malaria cases. However, for the mean proportion of patients tested with RDTs, there was a significantly smaller mean proportion of patients tested with RDTs compared to expected [80.3\% vs $92.2 \%$; RR 0.87 (0.78, $0.97)$ ]. In addition, there was a smaller mean proportion of malaria cases prescribed AL compared to expected, (89.1\% vs $94.4 \%$; RPR $=0.94(0.90,0.99))$. This was likely due to an increase in the percentage of patients with laboratory confirmed malaria that received a prescription of dihydroartemisinin-piperaquine (DP); this percentage rose from $0.04 \%$ in the pre-COVID- 19 period to 0.3\% from April 2020-September 2020 and to 3.7\% in the period from October 2020 through March 2021.

\section{Discussion}

This study evaluated the impact of the COVID-19 pandemic on the number of outpatient visits, malaria disease burden and case management practices between April 2020 and March 2021 using routine surveillance data collected at 17 high-volume, public, outpatient facilities in areas of varying malaria transmission across Uganda. In this study the number of outpatient visits, malaria cases, and non-malaria visits, as well as most indicators of malaria case management, did not reflect any significant changes in the first year following the onset of the COVID-19 epidemic. However, from October 2020 through March 2021, a modestly lower mean proportion of suspected malaria patients received an RDT for malaria diagnosis compared to what was expected for that period in the absence of COVID-19. Notably, this difference was not reflected by a change in the proportion of patients with suspected malaria who underwent diagnostic testing, as microscopy was available as an alternative to RDTs. A similar trend was observed in the last six months of observation for the mean proportion of malaria cases prescribed AL.

The emergence and rapid spread of COVID-19 across the world has created massive global disruptions on health systems, social services, and economic activity [20]. The disruption in health services is expected to be magnified in sub-Saharan Africa to a greater degree than in other regions due to relatively weak health service infrastructures, low clinician to population ratios, limited laboratory capacity, and a higher burden of other infectious diseases [21]. Delivery of malaria preventive measures and care services are some of the activities most likely to be affected by the epidemic given the high prevalence of malaria in the region [22]. Indeed, there are predictions that malaria cases will increase, and malariarelated mortality may nearly double, decelerating any gains attained in the last decade $[2,3,12,23]$. Uganda,

Table 2 Estimates of the impact of COVID-19 epidemic on disease burden during the first and second six months of the COVID-19 time period

\begin{tabular}{|c|c|c|c|c|c|c|}
\hline \multirow[t]{2}{*}{ Outcomes } & \multicolumn{3}{|c|}{ April 2020-September 2020} & \multicolumn{3}{|c|}{ October 2020-March 2021} \\
\hline & Observed $^{\mathrm{a}}$ & Expected $^{\mathrm{a}}$ & Ratio $[95 \% \mathrm{Cl}]^{\mathrm{b}}$ & Observed $^{\mathrm{a}}$ & Expected $^{a}$ & Ratio $[95 \% \mathrm{Cl}]^{\mathrm{b}}$ \\
\hline Total number of outpatient visits & 196,300 & 216,822 & $0.91[0.82,1.00]$ & 187,573 & 186,136 & $1.01[0.81,1.23]$ \\
\hline No of visits with a malaria diagnosis & 75,825 & 82,8842 & $0.92[0.76,1.14]$ & 60,032 & 50,050 & $1.20[0.75,1.84]$ \\
\hline Number of non-malaria visits & 120,475 & 123,902 & $0.97[0.92,1.04]$ & 127,541 & 118,890 & $1.07[0.95,1.21]$ \\
\hline$\%$ Suspected/visits & $61.9 \%$ & $64.1 \%$ & $0.97[0.93,1.04]$ & $61.6 \%$ & $61.9 \%$ & $0.99[0.89,1.22]$ \\
\hline \% Tested with RDT & $85.4 \%$ & $88.3 \%$ & $0.97[0.91,1.01]$ & $80.3 \%$ & $92.2 \%$ & $0.87[0.78,0.97]$ \\
\hline$\%$ Malaria cases prescribed AL & $94.2 \%$ & $95.2 \%$ & $0.99[0.98,1.00]$ & $89.1 \%$ & $94.4 \%$ & $0.94[0.90,0.99]$ \\
\hline
\end{tabular}

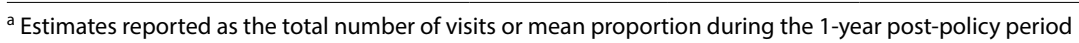

${ }^{b}$ For visit outcomes, this represents an incidence rate ratio; for proportions, a relative percent ratio. Assumed a constant population at-risk over the study period 
one of the highest malaria burden countries in sub-Saharan Africa, was predicted to be one of the countries most likely to be affected by the COVID-19 pandemic. According to a WHO modelling study, by the end of 2020 in a scenario where LLIN campaigns are not implemented and LLIN continuous distributions and access to effective anti-malarial treatment are reduced by up to $75 \%$, malaria deaths could increase by $200 \%$ in Uganda [12]. A study by Bell et al. predicted less disastrous effects resulting from a widespread COVID-19 outbreak compared to the impact of lockdowns on malaria programmes [24].

However, contrary to those worst-case scenarios, these data suggest that despite travel restrictions and other lockdown measures, access to care and malaria case management were largely unaffected by the COVID19 pandemic in the first year after it was confirmed in Uganda. This could be because $75 \%$ of the population in Uganda resides in rural areas where patients seeking care either walk or ride bicycles to the health facilities [25], making access to care possible even with restrictions to motor vehicle movements. In addition, the health system is structured in such a way that most of the health care staff are housed at or near the facilities; furthermore, travel permits were provided for health workers who needed to travel long distances to their duty stations, preventing the staff shortages that would likely have limited access to malaria care. Finally, planned malaria control activities, including the mass LLIN distribution campaign (implemented between June 2020 and March 2021) and IRS rounds, were successfully implemented despite the pandemic.

In addition, the outbreak was expected to increase fever cases because COVID-19 and malaria both present with fevers. However, community transmission of COVID-19 remained low during the first year following the outbreak; this may explain the fact that this analysis showed no increase in non-malarial visits or proportion of cases with suspected malaria. However, a decrease in the proportion of patients tested for malaria using RDTs was observed. This may be because of global supply chain issues that resulted in local stock-outs of RDTs. In the last year, there was a noted global decline in malaria RDT production and supply, with manufactures shifting in focus from RDTs to COVID-19 point-of-care tests $[26,27]$. Importantly, the decrease in proportion of RDTs used as a laboratory diagnostic did not affect whether or not patients received a diagnostic test for suspected malaria because microscopy was available as an alternative diagnostic at the MRCs.

There was also a modest decrease in the mean proportion of malaria cases prescribed $\mathrm{AL}$, the standard of care for the treatment of uncomplicated malaria in Uganda, seen in the last six months of observation. Given that a major limitation of interrupted time series analyses is that a change in a time series could be due to another factor that co-occurs with the intervention studied (in this case the arrival of the COVID-19 pandemic in Uganda), it was prudent to investigate whether there were any other changes affecting AL use at health facilities during the COVID-19 time period. Recently, Uganda's Ministry of Health secured funding to procure DP for use as second-line treatment for uncomplicated malaria in the event of treatment failure for patients using $\mathrm{AL}$, and for prescription to all patients with severe malaria following completion of three intravenous artesunate doses [28]. Therefore, from 2020 to-date, DP has been available at some public health facilities for use as a secondline treatment for patients with treatment failure due to $\mathrm{AL}$ and all patients diagnosed with severe malaria following completion of artesunate treatment, leading to an increase in the number of patients prescribed DP as described in the results. The decrease in prescriptions of AL observed in the last six months of the study may not be due to a decrease in supply or availability of AL due to the COVID-19 pandemic but instead due to changing prescription practices in response to policy changes regarding DP. There was no direct data on AL stock captured at these health facilities over the timeframe of the analysis, and as such this study was unable to conclusively attribute this decline in AL use to supply chain difficulties caused by the COVID-19 pandemic.

Other studies in similarly resource-restricted settings have shown varied impacts of the COVID-19 pandemic on malaria burden and deaths. A brief report published regarding patients at one health facility in Sierra Leone showed a decrease in the number of malaria cases diagnosed in children younger than five years of age in April 2020 compared to April 2019, but this had recovered by May 2020 [29]. However, this study was at a single health facility, did not account for seasonality, and only observed three months of the COVID-19 time period. A larger study in Zimbabwe, which utilized routine malaria surveillance data collected from all public and private health facilities from January 2017 to June 2020, found an excess of over 30,000 malaria cases from January to June 2020 compared to an average over the same period from 2017, 2018, and 2019 data [30]. This study also found that the number of malaria deaths recorded from January to June 2020 exceeded the annual totals for 2018 and 2019. However, while they describe these increases by comparing numbers from January 2020 to June 2020 to prior data, they do not make any statistical inference regarding their findings and note the limitations of routine surveillance data regarding data quality. A particular strength of the study presented here is the use of a quasi-experimental, interrupted time series study design which accounted 
for pre-COVID trends, other factors such as seasonality, rainfall, differences in study site, and concurrent LLIN and IRS campaigns, to predict unobserved counterfactual outcomes one year after the onset of COVID-19. In addition, the MRCs included had a full three years of preCOVID data that had undergone a supervised data cleaning process, resulting in less than $5 \%$ missing data.

Several limitations of this study are worth noting. Data from only 17 MRCs was used for this study because highquality data over a sufficient length of pre-intervention time are important to establish a baseline trend in a time series analysis, and most MRCs did not have three years of pre-COVID-19 data. The majority of the MRCs included in the study are in rural areas and, as seen in Fig. 1, are not evenly distributed throughout the country. Therefore, this study's findings may not be generalizable to the entirety of the country, especially to geographic areas not represented in the study and to more urban areas. Though similar outcomes are available through national health management information systems (HMIS) databases, HMIS data have been shown to suffer from increased 'missingness' and quality control problems $[31,32]$ and to provide robust estimates these data were not included. In addition, the impact of the COVID19 pandemic on malaria mortality was not assessed, as these data were not available. This study was also limited in its ability to assess what drugs patients actually took since data were only available on antimalarial prescription practices and not whether anti-malarial drugs were actually available and administered.

Importantly, COVID-19 cases were still relatively low in Uganda during the period this study was conducted, and it is likely that the impact of the COVID-19 pandemic on malaria indicators may change as community transmission increases, especially with the Delta variant now in the country. This is already being observed as the number of cases between March and July 2021 has doubled compared to what was observed between March and July $2020(90,656$ versus 40,962) [33]. Thus, the conclusions drawn in this study only reflect the effect of the COVID-19 pandemic on malaria indicators in the first year after arrival of COVID-19 in Uganda. The surge in cases caused by the Delta variant resulted in a second government-imposed lockdown in June 2021. Therefore, additional studies on the impact of the epidemic and the effect of lockdown policies will be required.

Finally, though ITSA is known to be a robust method of utilizing observational longitudinal data for estimating causal effects $[14,16]$, the study cannot rule out that estimates may have been subject to unmeasured confounding. For the AL outcome in particular, other health policy changes may be responsible for the decline in prescriptions of AL seen in the second six months of the COVID-19 time period. However, this does not change the central finding, which showed very little effect of the COVID-19 pandemic on malaria burden and case management in the first year after the arrival of COVID-19 in Uganda. Furthermore, if the decline in RDT use is confirmed to be due to supply chain issues and stock-outs, this is critical feedback for the Ministry of Health, so that plans can be made to source additional RDTs and/or to support the availability of microscopy at health facilities.

\section{Conclusion}

Utilizing routine surveillance data from 17 rural health facilities across Uganda, this study found the onset of the COVID-19 epidemic did not have major effects on malaria disease burden and indicators of case management, except for a modest decrease in the mean proportion of RDTs used for malaria diagnosis and the mean proportion of malaria cases prescribed AL in the second half of the COVID-19 year. However, continued surveillance will be essential to monitor for any changes in trends in malaria indicators due to the indirect effects of the COVID-19 pandemic so that necessary public health interventions can be instituted early to prevent increases in malaria morbidity and mortality. For example, now that a decrease in the usage of RDTs has been shown, it will be important to further investigate the cause of this decline and implement solutions by sourcing additional RDT supplies or ensuring access to high-quality microscopy at all public health facilities.

\section{Abbreviations \\ AL: Artemether-Lumefantrine; COVID: Corona virus disease; DP: Dihydroar- temisinin-piperaquine; HMIS: Health management information system; ICEMR: International Centre of Excellence in Malaria Research; IDRC: Infec- tious Diseases Research Collaboration; IRR: Incidence rate ratio; IRS: Indoor residual spraying; ITSA: Interrupted time series analysis; LLINs: Long-lasting insecticidal nets; $\mathrm{MOH}$ : Ministry of Health; MRCs: Malaria Reference Centres; RDTs: Rapid diagnostic tests; SARS-CoV-2: Severe acute respiratory syndrome Coronavirus 2; UMSP: Uganda Malaria Surveillance Project; WHO: World Health Organization.}

\section{Supplementary Information}

The online version contains supplementary material available at https://doi. org/10.1186/s12936-021-04018-0.

Additional file 1: Fig. S1. Observed mean proportion of patients with suspected malaria tested with a laboratory diagnostic over the study period. Vertical red line represents the start of the COVID-19 time-period on 1 April, 2020. Vertical black dashed line represents the 6-month midpoint of the COVID-19 time period (1 October, 2020).

Acknowledgements

We thank the study team and the Infectious Diseases Research Collaboration (IDRC) for administrative and technical support. 


\section{Authors' contributions}

JFN and JIN conceived of the study. JFN led the data collection activities with support from JIN, MK, GD, JO, AS, JKA, EA, CE, SGS, CMS, and MRK. MER and $J B$ led the data analysis with support from GD. JFN and JIN drafted the manuscript with support from JB and GD. JIN, JFN, MRK, AS, JKA, EA, SGS, JO, CE, BG, CMS, GD, and JB contributed to interpretation of the results and edited the manuscripts. All authors read and approved the final manuscript.

\section{Funding}

Funding was provided by the National Institute of Health as part of the International Centers of Excellence in Malaria Research (ICEMR) programme (U19AI089674) and the Makerere University Research and Innovations Fund (Ref: MAKRIF/DVCFA/026/20) under the RIF Award proposal titled "COVID-19 syndromic and sero-surveillance using established Uganda Malaria Surveillance sites". This work was supported in part by the Bill \& Melinda Gates Foundation grant INV-017894. Under the grant conditions of the Foundation, a Creative Commons Attribution 4.0 Generic License has already been assigned to the Author Accepted Manuscript version that might arise from this submission. JIN is supported by the Fogarty International Center (Emerging Global Leader Award grant number K43TW010365). EA and JFN are supported by the Fogarty International Center of the National Institutes of Health under Award Number D43TW010526. The funders had no role in the study design, data collection and analysis, decision to publish or preparation of the manuscript.

\section{Availability of data and materials}

The datasets generated and/or analysed during the current study are available at https://github.com/EPPIcenter/COVID_ITSA.

\section{Declarations}

\section{Ethics approval and consent to participate}

Ethical approval for study procedures and data collection was provided by the following ethics committees: School of Medicine College of Health Sciences at Makerere University (REF 2020-208), and Uganda National Council of Science and Technology (REF HS1125ES). Written informed consent was not required by the ethical review committees due to the routine, de-identified nature of the data.

\section{Consent for publication}

Not applicable.

\section{Competing interests}

None to declare.

\section{Author details}

${ }^{1}$ Infectious Diseases Research Collaboration, Kampala, Uganda. ${ }^{2}$ Department of Medicine, University of California San Francisco, San Francisco, CA, USA. ${ }^{3}$ Malaria Elimination Initiative, Institute of Global Health Sciences, University of California, San Francisco, San Francisco, CA, USA. ${ }^{4}$ National Malaria Control Division, Ministry of Health, Kampala, Uganda. ${ }^{5}$ Department of Medicine, Makerere University, College of Health Sciences, Kampala, Uganda. ${ }^{6}$ Department of Clinical Research, London School of Hygiene \& Tropical Medicine, London, UK.

\section{Received: 16 August 2021 Accepted: 8 December 2021}

Published online: 20 December 2021

\section{References}

1. WHO. World Malaria Report 2020. Geneva, World Health Organization, 2020. Accessed from: https://www.who.int/publications/i/item/97892 40015791 on 19 April 2021. 2020.

2. Weiss DJ, Bertozzi-Villa A, Rumisha SF, Amratia P, Arambepola R, Battle KE, et al. Indirect effects of the COVID-19 pandemic on malaria intervention coverage, morbidity, and mortality in Africa: a geospatial modelling analysis. Lancet Infect Dis. 2021;21:59-69.

3. Hogan $A B$, Jewell BL, Sherrard-Smith E, Vesga JF, Watson OJ, Whittaker C, et al. Potential impact of the COVID-19 pandemic on HIV, tuberculosis, and malaria in low-income and middle-income countries: a modelling study. Lancet Glob Health. 2020;8:e1132-41.

4. Sherrard-Smith E, Hogan AB, Hamlet A, Watson OJ, Whittaker C, Winskill P, et al. The potential public health consequences of COVID-19 on malaria in Africa. Nat Med. 2020;26:1411-6.

5. Ministry of Health. Overview of Malaria in Uganda (2014 - 2020). Accessed from: https://www.health.go.ug/programs/national-malar ia-control-program/\#: :text=Overview\%20of\%20Malaria\%20in\% 20Uganda\%20(2014\%20\%E2\%80\%93\%202020)\&text=Clinically\%20dia gnosed\%20malaria\%20is\%20the,20\%25\%20of\%20all\%20hospital\%20dea ths. on 29 July 2021.

6. Ministry of Health Uganda. Malaria Indicator survey (MIS) 2009. Accessed from: https://dhsprogram.com/methodology/survey/survey-display-332. cfm on 28 July 2021. 2009.

7. Ministry of Health. 2018-19 Uganda Malaria Indicator Survey. Acccessed from: https://dhsprogram.com/pubs/pdf/ATR21/ATR21.pdf on 19 April 2021. 2020

8. Ministry of Health. COVID-19 RESPONSE INFO HUB. Accessed from: accessed on 30th June 2021. 2020.

9. Yadav AK. Impact of lockdown to control over Novel Coronavirus and COVID-19 in India. J Family Med Prim Care. 2021;9:5142-7.

10. Wang S, Ye Y, Hu K, Lei H, Chen C, Xu X, et al. [Impact of Wuhan lockdown on the spread of COVID-19 in China: a study based on the data of population mobility](in Chinese). Zhejiang Da Xue Xue Bao Yi Xue Ban. 2021;50:61-7.

11. Lau H, Khosrawipour V, Kocbach P, Mikolajczyk A, Schubert J, Bania J, et al. The positive impact of lockdown in Wuhan on containing the COVID-19 outbreak in China. J Travel Med. 2020;27:taaa037.

12. WHO. The potential impact of health service disruptions on the burden of malaria: A modelling analysis for countries in sub-Saharan Africa. Geneva, World Health Organization. Accessed from: https://www.who.int/publi cations/i/item/9789240004641 on 15 April 2021. 2020.

13. Ashton RA, Bennett A, Al-Mafazy A-W, Abass AK, Msellem MI, McElroy P, et al. Use of routine health information system data to evaluate impact of malaria control interventions in Zanzibar, Tanzania from 2000 to 2015. EClinicalMedicine. 2019;12:11-9.

14. Bernal JL, Cummins S, Gasparrini A. Interrupted time series regression for the evaluation of public health interventions: a tutorial. Int J Epidemiol. 2017;46:348-55.

15. Kontopantelis E, Doran T, Springate D, Buchan I, Reeves D. Interrupted time-series analysis: a regression based quasi-experimental approach for when randomisation is not an option. BMJ. 2015;350:h2750.

16. Wagner AK, Soumerai SB, Zhang F, Ross-Degnan D. Segmented regression analysis of interrupted time series studies in medication use research. J Clin Pharm Ther. 2002;27:299-309.

17. Lopez Bernal JA, Gasparrini A, Artundo CM, McKee M. The effect of the late 2000s financial crisis on suicides in Spain: an interrupted time-series analysis. Eur J Public Health. 2013;23:732-6.

18. Sserwanga A, Harris JC, Kigozi R, Menon M, Bukirwa H, Gasasira A, et al. Improved malaria case management through the implementation of a health facility-based sentinel site surveillance system in Uganda. PLoS One. 2011;6:e16316.

19. Ministry of Health Uganda. Uganda Clinical Guidelines 2016: National Guidelines for Management of Common Conditions. Kampala; 2016.

20. United Nations Sustainable Development Group. Policy Brief: Impact of COVID-19 in Africa. Accessed from: https://unsdg.un.org/sites/default/ files/2020-05/Policy-brief-Impact-of-COVID-19-in-Africa.pdf on 15 April 2021. 2020.

21. El-Sadr WM, Justman J. Africa in the Path of Covid-19. N Engl J Med. 2020;383:e11.

22. WHO. World Malaria Report 2020. Geneva, World Health Organization, 2020. Accessed from: https://www.who.int/publications/i/item/97892 40015791 on 19 April 2021.

23. Aborode AT, David KB, Uwishema O, Nathaniel AL, Imisioluwa JO, Onigbinde SB, et al. Fighting COVID-19 at the expense of malaria in Africa: the consequences and policy options. Am J Trop Med Hyg. 2021;104:26-9.

24. Bell D, Hansen KS, Kiragga AN, Kambugu A, Kissa J, Mbonye AK. Predicting the impact of COVID-19 and the potential impact of the public health response on disease burden in Uganda. Am J Trop Med Hyg. 2020;103:1191-7. 
25. Uganda Ministry of Health. Uganda National Household survey 2016/2017 report. Kampala, 2016. Accessed from: https://www.ubos.org/ wp-content/uploads/publications/03_20182016_UNHS_FINAL_REPORT. pdf on 10 May 2021.

26. Global Fund. COVID-19 impact on health product supply: assessment and recommendations. The Global Fund Covid-19 Latest Information. 2020. https://www.theglobalfund.org/media/9440/psm_covid-19imp actonsupplychainlogistics_report_en.pdf

27. Nghochuzie NN, Olwal CO, Udoakang AJ, Amenga-Etego LN-K, Amambua-Ngwa A. Pausing the fight against malaria to combat the COVID19 pandemic in Africa: is the future of malaria bleak? Front Microbiol. 2020;11:1476.

28. Ministry of Health Uganda. Integrated Management of Malaria Training: Facilitators'Manual. Kampala, 2019. http://library.health.go.ug/publi cations/malaria/intergrated-management-malaria-training-facilitatorsmanual-third-edition

29. Buonsenso D, lodice F, Cinicola B, Raffaelli F, Sowa S, Ricciardi W. Management of malaria in children younger than 5 years old during coronavirus disease 2019 pandemic in Sierra Leone: a lesson learned? Front Pediatr. 2021;8:587638.

30. Gavi S, Tapera O, Mberikunashe J, Kanyangarara M. Malaria incidence and mortality in Zimbabwe during the COVID-19 pandemic: analysis of routine surveillance data. Malar J. 2021;20:233.

31. M \& E Technical Support Programme. Status and Strategies for the Utilization of the Health Management Information System (HMIS) in Uganda 2018 [Available from: https://mets.or.ug/health-management-infor mation-system-hmis-in-uganda/.

32. Kagoya HR, Kibuule D. Quality assurance of health management information system in Kayunga district, Uganda. Afr Evaluation J. 2018;6:a238.

33. WHO. Health Emergency Dashboard n.d. Geneva, World Health Organization. [Available from: https://covid19.who.int/region/afro/country/ug.

\section{Publisher's Note}

Springer Nature remains neutral with regard to jurisdictional claims in published maps and institutional affiliations.

- fast, convenient online submission

- thorough peer review by experienced researchers in your field

- rapid publication on acceptance

- support for research data, including large and complex data types

- gold Open Access which fosters wider collaboration and increased citations

- maximum visibility for your research: over 100M website views per year

At BMC, research is always in progress.

Learn more biomedcentral.com/submissions 